\title{
La psicoterapia en la transformación de la praxis moral y las actitudes hacia la paz Investigación de realismo crítico
}

\author{
Mikel Garcia Garcia ${ }^{1,2,3,4}$ \\ ${ }^{1}$ Médico. Psicólogo. Psicoanalista junguiano. \\ ${ }^{2}$ Cursando doctorado en Estudios Internacionales de Paz, Conflictos y Desarrollo en la \\ Universitat Jaume I (UJI Castellón) \\ ${ }^{3}$ Miembro de la Sociedad Internacional para el desarrollo del Psicoanálisis Junguiano. \\ http://sidpaj.es/ \\ ${ }^{4}$ Psicoterapeuta acreditado por FAPyMPE - Federación de Asociaciones de Psicólogos y \\ Médicos Psicoterapeutas de España \\ Correspondencia iratxomik@gmail.com
}




\section{Resumen}

Aunque existe evidencia de que la psicoterapia transforma al paciente, no existe suficiente información sobre cambios en su praxis moral ni en sus actitudes hacia la paz. Este artículo informa de una investigación cuyo objetivo principal fue conocer esa realidad. Otro objetivo era explorar asociaciones entre datos recogidos. Diseño: marco epistemológico de realismo crítico con un análisis cualitativo -Grounded Theory-, y cuantitativo para la acción participativa. Una primera fase inductiva analizando respuestas cualitativas para llegar a construir una teoría. Una segunda fase deductiva para verificarla con hipótesis nulas iniciales para el análisis de datos cuantitativos. Método: La muestra se captó principalmente por internet. Datos recogidos mediante cuestionarios: sociodemográficos; de personalidad mediante el MCMI; de dilemas éticos; de disposición a la paz. Los dilemas, con respuestas dicotómicas y descriptivas, y el cuestionario de paz se diseñaron para la investigación. Muestra válida: 85 sujetos. Análisis: Cualitativo mediante el programa Atlas.ti, y cuantitativo mediante el programa SPSS. El análisis cualitativo genera teoría: La imagen de la víctima es una personificación del modo en que un sujeto se imagina a sí mismo sufriendo. Se formulan hipótesis nulas a verificar cuantitativamente. $\mathrm{H}_{0}$ : Realizar como paciente una psicoterapia no cambia la disposición moral. H01: Tampoco aumenta la disposición a la paz. Resultados: a) se rechazan las hipótesis nulas. Hacer psicoterapia cambia las elecciones morales incrementando las utilitaristas y aumenta la disposición a la paz; b) a más edad más utilitarismo; c) las personalidades compulsivas con sentimiento de culpa hacen elecciones deontologistas. Discusión: La moral deontológica tiene una función contenedora de acciones morales asociales. Conclusiones: La articulación cuantitativo cualitativo, poco frecuente en las investigaciones, ha evidenciado potencial generador de significado. Es importante un trabajo de elaboración moral antes del alta de los pacientes. La investigación es incompleta y anticipa áreas a tener en cuenta en futuros trabajos.

Palabras clave: dilemas morales; psicoterapia; desarrollo moral; consiliencia

\section{Abstract:}

Although there is evidence that psychotherapy transforms the patient, there is not enough information about changes in their moral praxis or in their attitudes toward peace. This article reports on an investigation whose main objective was to know that reality. Another objective was to explore associations between collected data. Design: epistemological framework of critical realism with a qualitative analysis -Ground Theory-, and quantitative for participatory action. A first inductive phase analyzing qualitative answers to get to build a theory. A second deductive phase to verify it with initial null hypotheses for the analysis of quantitative data. Method: The sample was mainly captured on the internet. Data collected through questionnaires: sociodemographic; of personality through the MCMI; of ethical dilemmas; of disposition to peace. The dilemmas, with dichotomous and descriptive answers, and the peace questionnaire were designed for research. Valid sample: 85 subjects. Analysis: Qualitative through the Atlas.ti program, and quantitative through the SPSS program. Qualitative analysis generates theory: The image of the victim is a personification of the way in which a subject imagines himself suffering. Null hypotheses to be quantitatively verified are formulated. HO: Performing a psychotherapy as a patient does not change the moral disposition. H01: Nor does the willingness to peace increase. Results: a) the null hypotheses are rejected. Doing psychotherapy changes moral choices by increasing utilitarians and increases disposition to peace; b) to more age plus utilitarianism; c) compulsive personalities with feelings of guilt make deontological choices. Discussion: Deontological morality has a content function of asocial moral actions. Conclusions: The qualitative quantitative articulation, rare in the investigations, has shown a potential generator of meaning. It is important a work of moral preparation before the discharge of patients. The research is incomplete and anticipates areas to be considered in future work.

Keywords: moral dilemmas; psychotherapy; moral development; consilience 


\section{Introducción}

Todos los síntomas psicopatológicos, aunque alguno más claramente (cleptomanía), se asocian a conductas amorales. En las terapias psicoanalíticas se constata que el analizando transforma su estructura cognitiva junto a la extinción de los síntomas clínicos. La extinción de la patología dota al sujeto, al liberarlo del determinismo, de un potencial de funcionamiento moral distinto. Aunque el psicoterapeuta dispone de criterios evaluativos para apreciar la capacitación del paciente para una praxis moral más autónoma, no es habitual evaluar objetivamente la cognición moral en las altas terapéuticas. En los seguimientos posteriores al alta a veces se recogen datos que ponen en duda que, en situaciones reales de la vida, las decisiones morales sean distintas. Clarificar este fenómeno requiere investigación en un marco distinto del clínico.

Kohlberg considera que es esencial comprender la estructura del razonamiento que evalúa los dilemas morales. En sus investigaciones son los aspectos formales del pensamiento moral los que interesan a Kohlberg, es decir, las razones que tienen las personas para elegir una u otra acción (Kohlberg, 1982; Kohlberg, 1984; Kohlberg, Power, \& Higgins, 1997). Hay dos posiciones contrastadas en el entendimiento clásico de la moralidad. La posición deontológica postula que ciertas acciones son siempre amorales, independientemente de cuán buenas sean las intenciones o resultados y que las decisiones morales se basan en respuestas innatas, automáticas, intuitivas y más emocionales (Kant, 2008; Scanlon, 1982, 1998). La posición utilitarista o consecuencialista (Mill, 1989; Regan, 1980; Elster \& Grapes, 1982; Bentham, 1983) postula que la acción correcta es la que provoca un bien mayor, independientemente de los medios utilizados y que las elecciones se basan en un razonamiento de deliberación evaluativa más lenta que conlleva esfuerzo. Un acto, por tanto, se juzga moralmente sólo por su resultado y consecuencias teniendo en cuenta la cantidad total de bien producido. Una situación típica de elección utilitarista es sacrificar un sujeto para salvar un grupo. Alasdair Maclntyrel, principal exponente de la nostalgia comunitarista, en After virtue (Maclntyre, 1981) hace un lúcido estudio histórico de la ética que concluye en un diagnóstico pesimista: la ética ya no es posible puesto que no es posible llegar a acuerdos morales ni fundamentarlos racionalmente. Cada sujeto se adscribe a una u otra forma de ética según la coherencia entre sus atractores internos y las ofertas externas: sus elecciones son, pues, relativistas. Cercana a esa posición relativista, más intersubjetiva, está la teoría diádica (Gray, Waytz, \& Young, 2012) que proporciona un marco en el que la cognición moral probablemente no se caracteriza por el conflicto entre las acciones de agentes y los resultados en pacientes, sino por su activación mutua. Teoría también cuestionada por investigaciones (Baxter, 2016) que apuntan a una prevalencia de la percepción de la mente. Para Kant los seres humanos nos caracterizamos por una insociable sociabilidad (Martínez Guzmán, 1997). Haidt y Joseph (2007) intentaron identificar las bases psicológicas mediante las cuales las diferentes culturas crean la gran variedad de sistemas morales. Encontraron cinco: Daño, Justicia y equidad, Filiación y lealtad, Autoridad y respeto, Pureza y santidad. Hay investigaciones 
controvertidas de la neurociencia. Algunas (Joshua D. Greene, Nystrom, Engell, Darley, \& Cohen, 2004) encuentran diferencias entre las evaluaciones morales de pacientes con daño en la Corteza Prefrontal Ventromedial -CPV y las personas sin daño neurológico. La hipótesis del paciente utilitarista (de moda en la neurociencia cognitiva), asocia lesión en CPV con mostrar una disposición utilitarista en sus respuestas a los dilemas morales personales revelando una posible carencia de empatía. Hay pocos estudios basados en rasgos de personalidad. Una realizada con psicópatas reveló que un subgrupo de estos (con poca ansiedad) hacía elecciones claramente utilitaristas (Koenigs, Kruepke, Zeier, \& Newman, 2012). En la recensión del libro de Darcia Narváez (2014) publicada en Anuari de Psicologia (Cunyat Agut, 2016), se destaca la vital importancia que para la moral tiene la experiencia temprana de los sujetos para la integración neurobiológica del cerebro triúnico de McLean quien propone en 1952 que el cerebro humano es en realidad tres cerebros en uno: el reptiliano, el sistema límbico y el neocórtex (MacLean, 1990).

De esta revisión se infiere que hay más literatura sobre la discusión entre deontologistas y utilitaristas, que sobre la intersubjetividad; y que la posición utilitarista se asocia a lesiones, a psicopatías... También se apunta que la mayor parte de las personas suelan preferir la opción deontologista, lo que indicaría que esta opción ha sido favorecida por la selección natural que busca maximizar la cooperación.

Esta investigación explora el funcionamiento moral con una perspectiva consiliente aportando datos acerca de la estructura de la personalidad y con una metodología de análisis cualitativo cuantitativo, que vas más lejos de la mirada reduccionista exclusivamente cuantitativa. La diferencia entre lo cuantitativo y lo cualitativo es poco precisa (Mayntz, Holm, Hübner, \& Muñiz, 1969). Resulta imposible que un investigador pueda recurrir a una forma sin utilizar la otra. El Enfoque Integrado Multimodal, también insiste en la integración de lo cuantitativo y cualitativo (Henwood, 2004, 2008).

Estas consideraciones innovan respecto a investigaciones previas. Esto puede ser relevante para la comunidad científica y para la comunidad clínica.

\section{Objetivos}

El objetivo principal es verificar si hay cambios en la estructura moral y de paz de los pacientes de psicoterapia y si los hay comunicar los hallazgos a terapeutas y comunidad científica. Otro objetivo es exploratorio para encontrar asociaciones entre variables que permitan seguir investigando.

\section{Material y método}

\section{Participantes.}

La muestra procede de dos vías: a) contacto directo con participantes de actividades sociales como seminarios, conferencias; b) mediante internet. El La psicoterapia en la transformación de la praxis moral y las actitudes hacia la paz. Mikel Garcia Garcia 
proyecto de investigación se publicó en redes sociales. Las muestras de internet son frecuentemente usadas en la investigación psicológica ya que mantienen la confiabilidad igual a las poblaciones basadas en laboratorio mientras que proporcionan una mayor diversidad (Skitka \& Sargis, 2006). Los sujetos participaron voluntariamente y sin remuneración (el sistema MTurk si remunera). Total 499 protocolos, el $66 \%$ captado en internet.

\section{Instrumentos}

Los participantes rellenaron un material. La mayoría lo hizo electrónicamente mediante formularios docs depositados en un espacio google. Secciones del material: a) sociodemográfica; b) religión; c) cuestionario de personalidad MCMI (Inventario Clínico Multiaxial de MILLON, 2017); d) Cuestionario de Paz. Construido por el investigador basándose en autores. Cuestionario de Paz o Equilibrio en La Vida (CPEV20) (Moral De la Rubia, Medina, \& Bravo, 2011); CONFLICTALK. Cuestionario de estilos de mensaje en el manejo del conflicto. (Kimsey \& Fuller, 2003); LAEA; Capacidad de resolución positiva del conflicto Conflict Scale EAC (Jenkins \& Smith, 1987) (2008); Inventario Balanceado de Respuestas Socialmente Deseables (Moral de la Rubia, García-Cadena, \& Antona-Casas, 2012; Paulhus, 1988); Listado de adjetivos autoconcepto (Garaigordobil, 2011). En el apéndice 1. Hay un listado de las variables medidas. e) ocho dilemas morales con dos respuestas una obligatoria (si/no) y otra voluntaria consistente en relatar las razones, los sentimientos, reflexiones, que le producía el dilema... El apéndice 2 contiene los dilemas usados en la investigación en el orden en que se presentaban. Se han excluido del estudio dilemas diseñados para explorar actitudes como la confianza, la lealtad invisible y la paranoia.

En su diseño se controlaron varias variables: a) orden de presentación; b) tipo de material; c) que recogieran aspectos que se discuten en las investigaciones sobre la moralidad; d) que abordasen situaciones factibles de estar cercanas a la experiencia de la gente en la actualidad y e) que promovieran contacto emocional y reflexión introspectiva en los participantes. Los dilemas tienen una naturaleza paradójica que los hace evocadores emocionalmente (J D Greene, Sommerville, Nystrom, Darley, \& Cohen, 2001).

¿Qué áreas exploran?

El dilema del moribundo la dicotomía personal Deontologismo/Utilitarismo. Este es el principal en la investigación. Se presenta en tercer lugar y mediante una imagen, para que emerja en un contexto de impacto emocional por los anteriores y convoque una respuesta inmediata sin el freno de tener que comprender un texto.

El dilema gratificación obliga a elegir una modalidad placentera en un contexto subyacente de dimensiones polares: intima/social y secreto/divulgación.

El dilema demanda de dolor sexual, confronta al sujeto con actuar un daño consentido. Un modo de dicotomía Deontologismo/Utilitarismo indirecta o impersonal.

El dilema perdón confronta con actuar desde el resentimiento o desde la compasión. En Libertad y resentimiento Strawson (1995) diserta acerca de ello.

El dilema autorizar inseminación confronta con la responsabilidad de sancionar el deseo de otro que demanda con cierta propiedad respecto a un tercero muerto. 
El dilema ecológico confronta el altruismo con el propio interés.

El conjunto contiene los factores recogidos por Haidt y Joseph.

Criterios de exclusión. Se han excluido los protocolos que no estaban totalmente cumplimentados y los que no cumplían los criterios de validez y fiabilidad del MCMI, resultando una muestra válida de 85 sujetos (17\%).

\section{Procedimiento}

Categorizaciones de la muestra.

1.- Basándose en el dilema del moribundo y contrastando la descripción abierta con su respuesta si/no, se ha codificado los sujetos como pertenecientes a una de estas tres categorías: deontologistas; utilitaristas y relativistas. La relativista es equivalente a la Utilitarista-Deontologista de la investigación de Everett et al (2016). Quedando la distribución: deontologistas $(61,2 \%)$, utilitaristas $(28,2 \%)$ y relativistas $(10,6 \%)$. La categoría relativista ha procedido sobre todo de los deontologistas según la respuesta dicotómica (48\% responden no tirar al agua al moribundo), y esto ha ocurrido más con las mujeres (55\%). Las mujeres son más relativistas (32\%).

2.- Con las respuestas de la sección de religión, se ha codificado los sujetos como pertenecientes a una de estas categorías: ninguna religión; espiritualidad; ateo/agnóstico; budista; monoteísta.

3.- Según el resultado de la corrección del MCMI se ha codificado los sujetos como pertenecientes a una de estas categorías: sano; neurótico; fronterizo; psicótico.

La categorización genera tres nuevas variables discriminativas a incluir para el análisis cuantitativo que se ha realizado mediante el programa estadístico SPSS.

\section{Análisis de datos}

Del total de la muestra

El análisis inicial sigue la metodología de la Grounded theory (GT) o teoría fundamentada. Una metodología sistemática que implica la construcción de teoría mediante el análisis de datos cualitativos. Prevalece la inducción, en contraste con el enfoque hipotético-deductivo. El método fue desarrollado por dos sociólogos, Barney Glaser y Anselm Strauss. Su colaboración en la investigación sobre pacientes hospitalarios moribundos los llevó a escribir Awareness of Dying en 1965. La teoría fundamentada combina diversas tradiciones en sociología, positivismo e interaccionismo simbólico que es metodológicamente dinámico. Para Glaser (2005), la estrategia de Grounded Theory es estudiar la interrelación entre el significado en la percepción de los sujetos y su acción. Las personas actúan sobre los objetos de su mundo e interactúan con otras personas a partir de los significados que los objetos y las personas tienen para ellas. Es decir, a partir de los símbolos.

Se ha utilizado la herramienta Atlas.ti para el análisis cualitativo con el fin de descubrir las redes semánticas subyacentes en las respuestas explicativas de todos los dilemas morales propuestos en la investigación y construir una teoría explicativa sin pretensión de verdad pero que cumpla criterios de ajuste, relevancia y viabilidad (Glaser y Strauss 1967). 
La unidad de análisis son los incidentes que son seleccionados de las descripciones, a partir de los cuales se construyen las categorías que se manifiestan a través de los datos. El ajuste entre categorías y los incidentes que están representando, depende de que comparación de incidentes con conceptos sea exhaustiva.

El investigador que utiliza esta metodología, tiene experiencia profesional y personal y saber en el área en estudio. Una sensibilidad teórica que le permite tener insights acerca de los fenómenos que se le aparecen y desarrollar la habilidad de entender, de reconocer y dar significado a los datos. Sin embargo, no debe partir de una a priori sino ir construyendo teoría a partir de los datos. El método es circular, el investigador examina los datos y empieza su codificación, categorización, conceptualización y escribe sus primeras reflexiones, cambiando el foco de atención y buscar otra dirección, según nuevos datos que van entrando en escena hasta que los datos empiecen a repetirse, y se llegue a saturación teórica.

\section{Resultado semántico}

La red tirar al moribundo tras el análisis mediante Atlas.ti. (ver Figura 1)

La elección de los deontologistas es no tirar al moribundo y está: 1. determinada por una espiritualidad asociada a no tener la potestad de controlar el destino; 2. causada por no poder soportar en el futuro el sentimiento de culpa y por la identificación con el moribundo especialmente si es conocido o familiar; 3. favorecida por la presión del grupo; 4. asociada a una actitud de sacrificio por ejemplo tirándose el sujeto al agua para dejar sitio al moribundo; 5. asociada a acompañar al muriente en su proceso de muerte cuidándolo; 6 . asociada a la esperanza en que ocurra algo que salve la situación, 7. asociada a la necesidad de que la decisión sea consensuada por el grupo; 8. asociada con el canibalismo, consistente en no tirar al moribundo y tenerlo como reserva para poder comerlo si el naufragio dura y no son rescatados.

La elección de los utilitaristas es tirar al agua al moribundo y se caracteriza por: 1. no estar impedida por el hecho de ser conscientes de que ese acto sería un suceso que les causara culpa en el futuro; 2 . estar asociada con sentimiento de dolor por la decisión; 3. estar asociada con indagar si hay otros candidatos en condiciones iguales o peores; 4 . asociada con la identificación y actitud de acompañamiento al moribundo; 5. el sacrificio es parte de esa actitud, uno siente que sacrifica algo. La elección de los relativistas es tirar al agua al moribundo y está asociada con: 1. hacerlo a un modo de eutanasia; 2 . tener una actitud ecológica; 3 . con una actitud caníbal. 


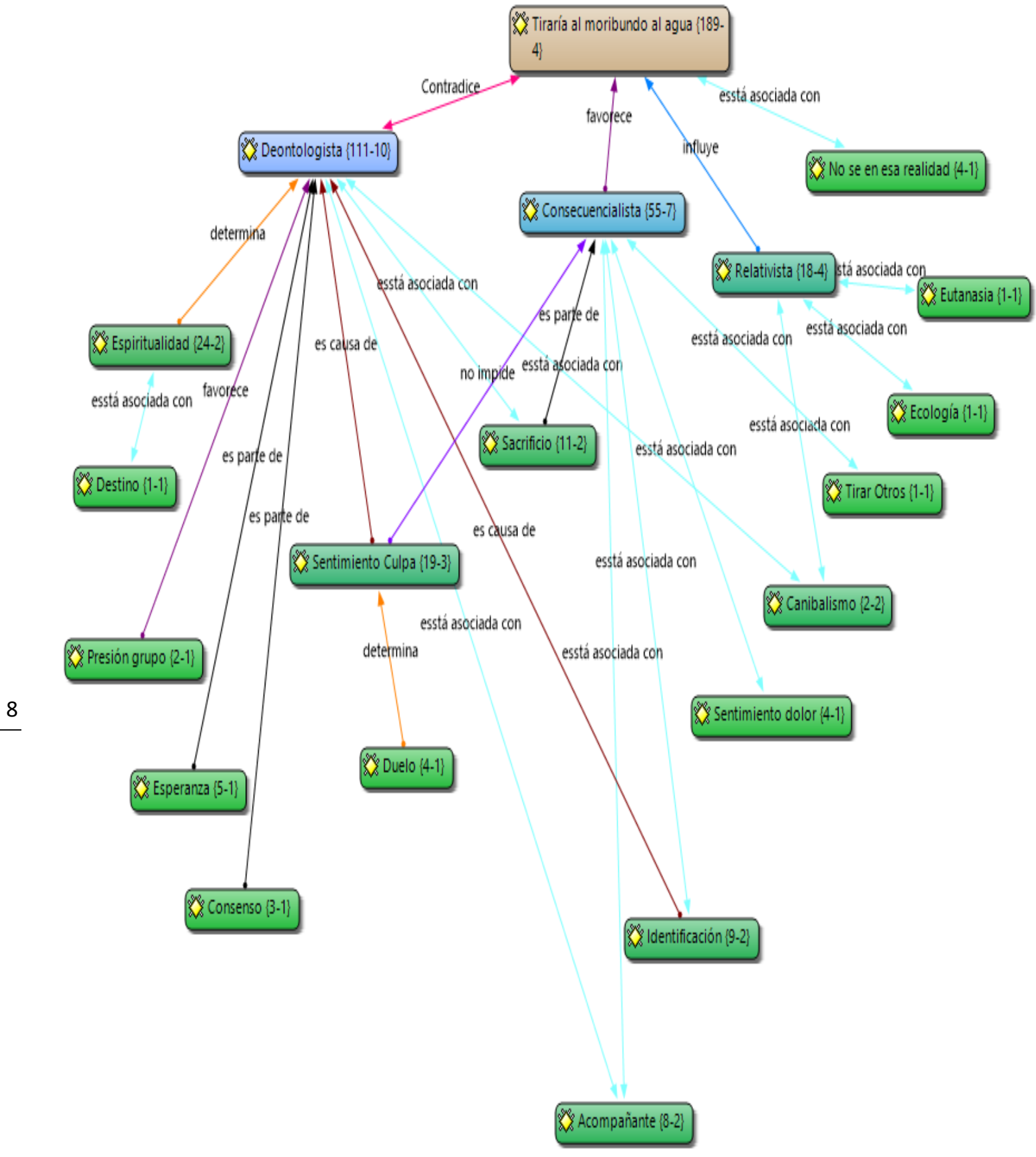

Figura 1 Red Tirar al moribundo Atlas.ti Clasificación topológica. Se indican las relaciones y los tipos de asociación de los conceptos codificados: asociación; determinación; favorece; influye... Los números entre paréntesis indican etiquetas en las categorizaciones del Atlas.ti 
La red semántica es el resultado de comparar incidentes, integrar categorías, reconocer relaciones, llegando a una inducción analítica de una teoría que es la construcción de una explicación acerca de un fenómeno.

El moribundo es percibido como una víctima. La imagen de la víctima que tiene un sujeto es una personificación del modo en que se imagina a sí mismo sufriendo. Esta imagen condiciona la decisión de tirar o no al agua al moribundo. Las dimensiones que más influencia tienen son el sacrificio, la empatía y la culpa. La relación entre dimensiones es compleja y detecta un factor conector explicativo que pertenece al orden de la personalidad del sujeto.

\section{Hipótesis para el análisis cuantitativo}

Formular una hipótesis desde el inicio promueve rigurosidad en la investigación cuantitativa. Formulo las hipótesis nulas siguientes teniendo en cuenta los resultados de la red semántica descrita. $\mathrm{H}_{0}$ : Realizar una psicoterapia no cambia la disposición moral del paciente hacia elecciones utilitaristas. $H_{01}$ : Tampoco aumenta la disposición a la paz.

\section{Análisis cuantitativo}

Los que no pertenecen a ningún tipo de religión son más relativistas. Los budistas son los más deontologistas. Los católicos se reparten entre los tres tipos de moral (ver Figura 2)

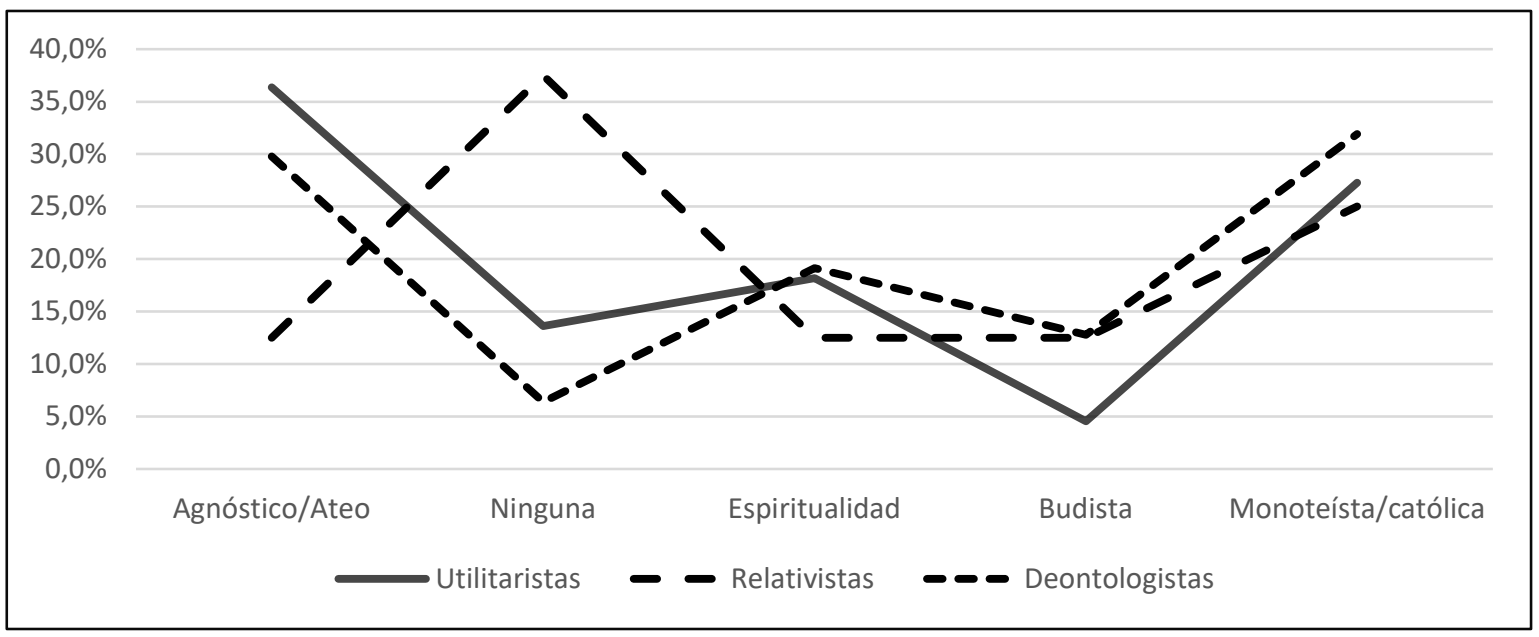

Figura 2 Religiosidad y elecciones morales

En la Figura 3 se representan las variables del cuestionario de paz que discriminan significativamente los utilitaristas de los deontologistas en función de sus puntuaciones medias.

Los utilitaristas tienen un perfil de paz mejor que la media poblacional y que los deontologistas. Respecto a estos últimos se diferencian significativamente (análisis de diferencia de medias t student) en no depender de 
la deseabilidad social, sin autoengaño inconsciente ( $s=0,002)$, manejar los conflictos centrándose en el problema $(s=0,015)$, teniendo una buena capacidad de resolución positiva del conflicto $(s=0,023)$ y mejor autoconcepto emocional ( $s$ $=0,033)$ e intelectual $(s=0,033)$.

Los deontologistas presentan un nivel de autoengaño inconsciente que pudiera estar relacionado con un conflicto entre los códigos deontológicos y los contextos de escenarios de los dilemas. Podrían tener una disposición a saltar el código, pero dado que la consecuencia sería un malestar y culpa, la defensa es autoengañarse aferrándose al principio deontológico.

10

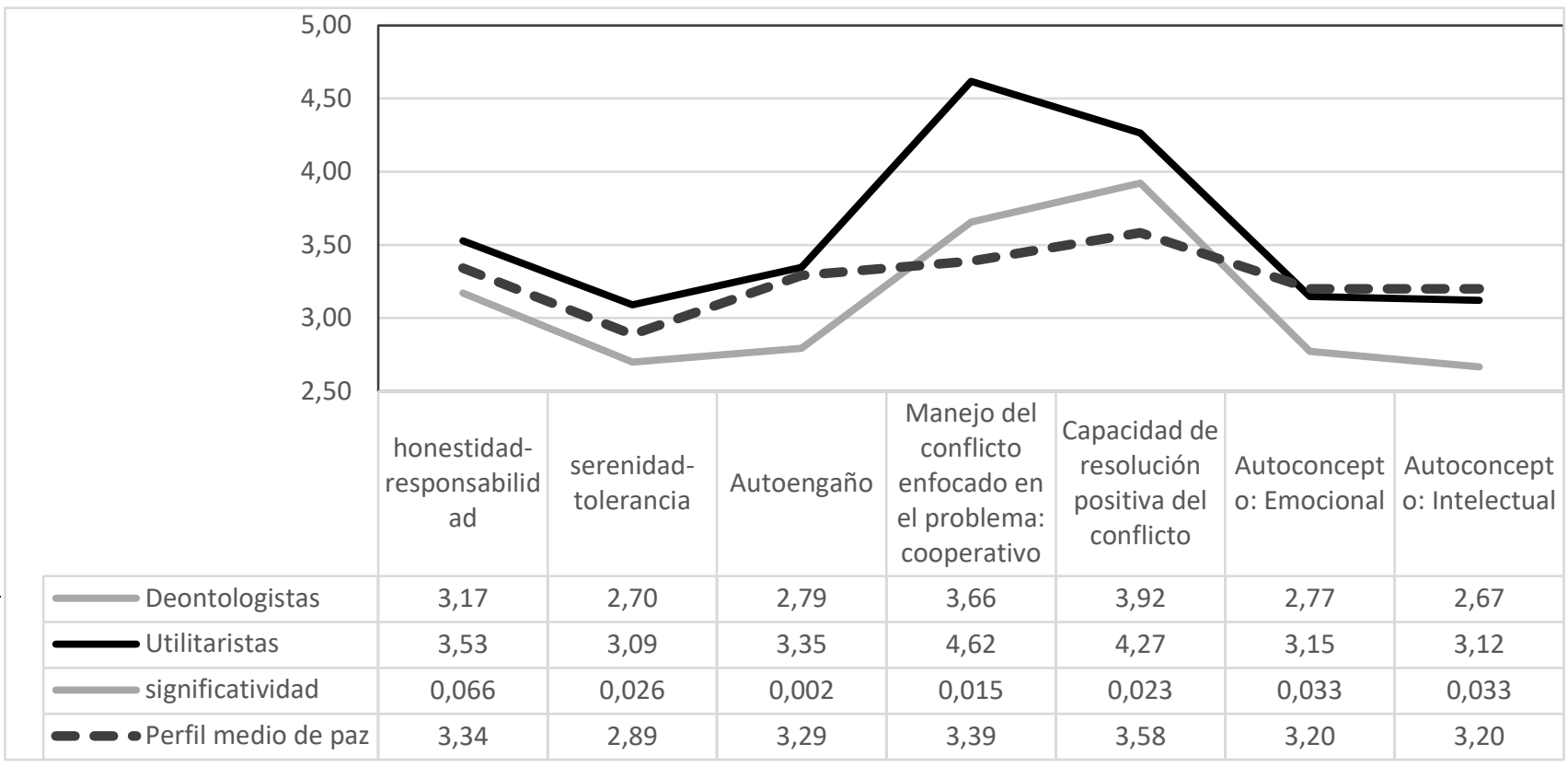

Figura 3 Medias y significatividad de diferencia de medias con t Student

Análisis de los datos de personalidad. La fiabilidad de las escalas del MCMI, es buena (alpha de Cronbach 0,82). Los análisis factoriales MCP han podido hacerse al cumplir los requisitos RMO $(0,829)$ y Bartlett (sig. 0.001).

Los utilitaristas en relación a los deontologistas tienen un perfil más saludable puntúan más en los patrones de personalidad más sanos y menos en las escalas clínicas, encontrándose un perfil opuestos en distimia, hipomanía, alcoholismo y drogadicción. La puntuación en distimia tiene significatividad estadística en el análisis t -test para grupos independientes $(\mu 1 \neq \mu 2)$. $[t(59)=2.45$, $p=.0312]$ (ver Figura 4).

En la Figura 5 se representa en perfil completo del MCMI de los sujetos deontologistas que explicaron que la culpabilidad que sentirían en el futuro su razón para no tirar al moribundo era. Este perfil se caracteriza por un patrón de personalidad compulsivo-esquizoide-evitante con depresión-ansiosa y contenciones sin compensaciones escapatorias como drogas o alcohol. 


\section{Gráfica de escalas que discriminan los tipos morales en el primer factor}

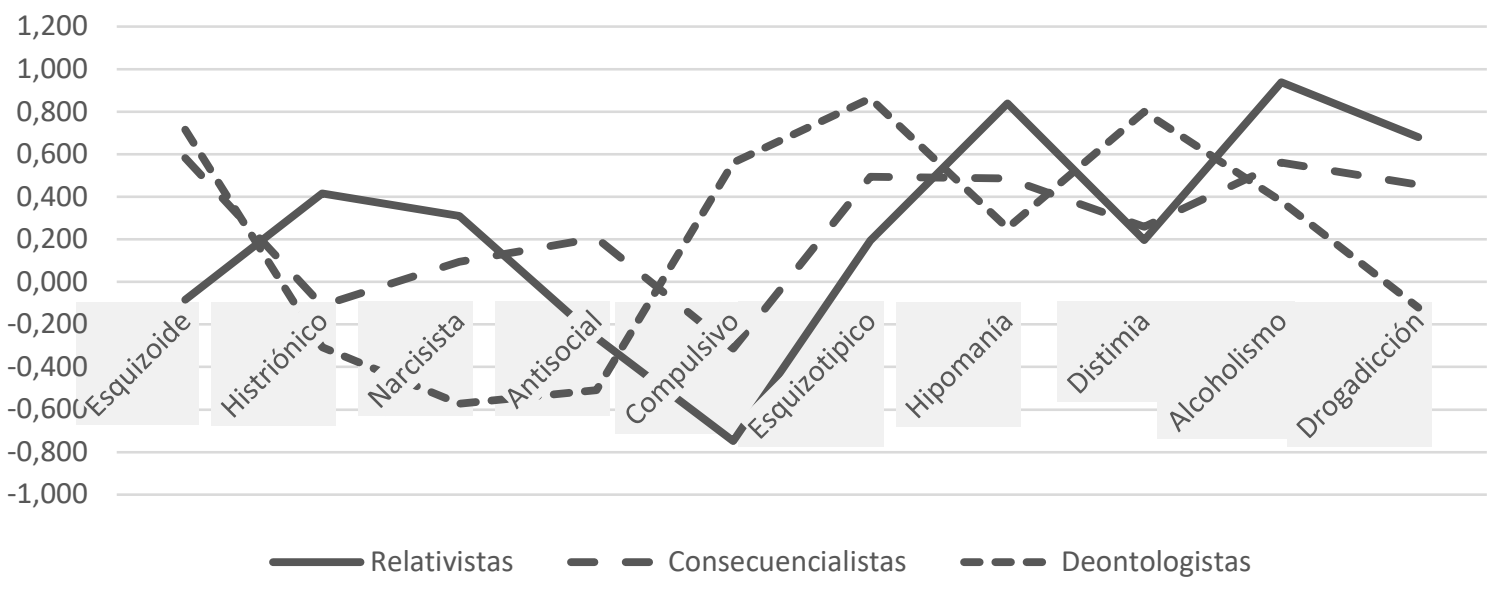

Figura 4 Escalas que discriminan los tipos morales en el primer factor

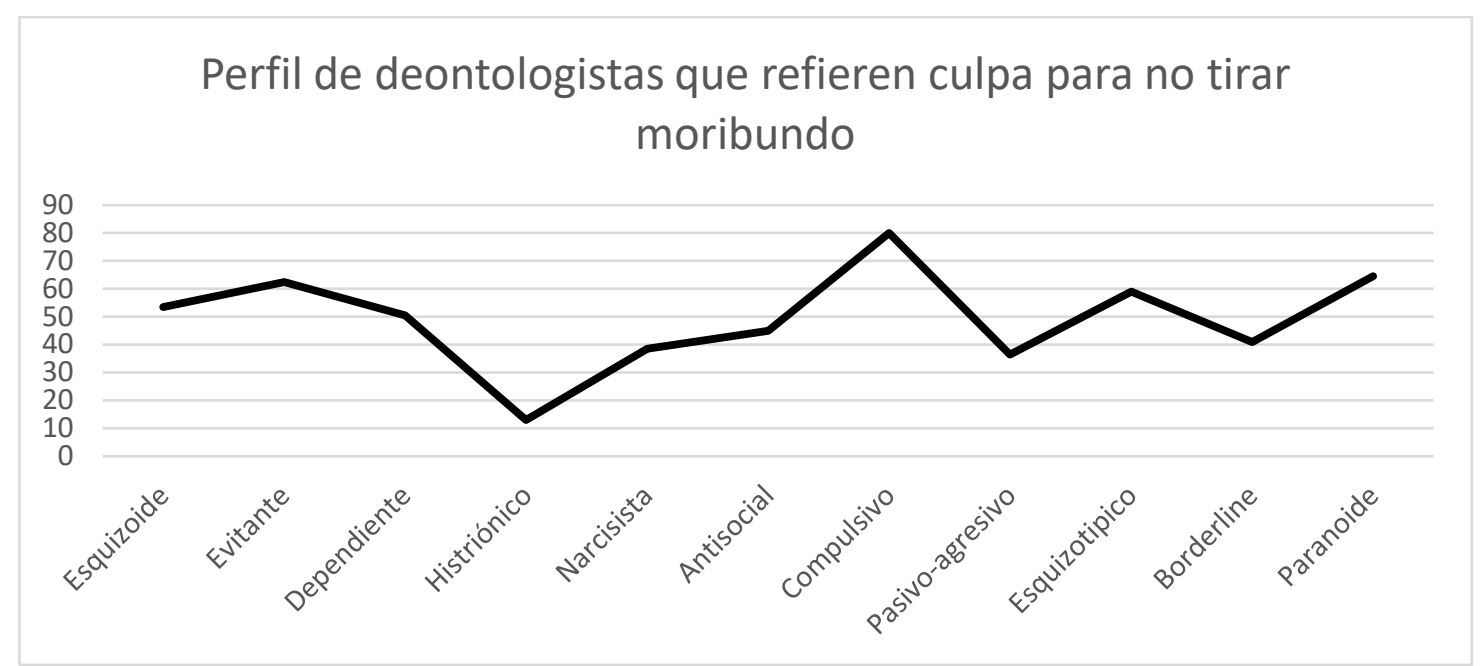

Figura 5 Escalas MCMI de los deontologistas que refieren culpabilidad

Los utilitaristas tienen más edad, con significatividad estadística. El test $(t(74)=3,496, p=.001)$. El F-test ANOVA de una vía $(F(84)=6,341, p=.003)$. Para $M=48,38$ años con un SD (+/-10,842), $M=39,19$ años con un SD (+/- 10,53).

\section{Análisis cuantitativo en relación a la psicoterapia.}

Los que han hecho psicoterapia, son los más utilitaristas $(47,10 \%)$ frente al $29,40 \%$ de los deontologistas, siendo esta diferencia estadísticamente significativa en el análisis Chi Cuadrado. $\left.\left[\chi^{2}(2, N=85)=9,533, p=.009\right)\right]$. Este subgrupo es el $20 \%$ del total de la muestra. Es una representación alta comprando con la población general. Según los datos de Bloomberg (2015) solo un 2,04 por ciento de la población había buscado ayuda psiquiátrica en los 12 meses estudiados, aunque la actividad de la psiquiatría española es más alta que en otros países. 
El análisis mediante regresión lineal, de las variables de personalidad del MCMI que pueden influir en el tipo de elección moral no ha encontrado asociaciones significativas.

El análisis de regresión para datos categóricos (escalamiento óptico) testando como predictores psicoterapia, edad y género, si ha encontrado significancia estadística [ANOVA $(F(84)=2,853, p=.011)$ ]. La psicoterapia $(F=7,76)$ y la edad $(3,192)$ influyen significativamente en la elección utilitarista.

\section{Resultados}

A) se rechazan las hipótesis nulas. Por lo tanto, hacer psicoterapia si determina un cambio hacia posiciones utilitaristas y hacia tener mayor disposición a la paz; B) los sujetos de más edad son los más utilitaristas; C) las personalidades compulsivas con sentimiento de culpa hacen elecciones deontologistas.

El trabajo de introspección y elaboración de la psicoterapia genera autoconocimiento, adquisición de un estilo de investigar sobre la conciencia y la capacidad de distanciarse de los automatismos internos.

Esas son condiciones para poder deliberar sobre el escenario del dilema y propicia elección utilitarista. Las descripciones ante los dilemas, analizadas cualitativamente, que aportan los sujetos manifiestan esa deliberación. El proceso de elección es complejo, contempla más dimensiones, es intersubjetivo. Varios afirmaron que sería doloroso tomar la decisión de tirar al moribundo y que ese dolor duraría tiempo o toda la vida. El proceso de psicoterapia acerca a ambos géneros, aunque sin significatividad estadística, hacia estilos más igualitarios de respuestas. Los de más edad destacan por hacer elecciones utilitaristas, dato probablemente influido por la dimensión temporal de la duración de la terapia de los sujetos que son más viejos cuando terminan. Podría deducirse que la mayor experiencia vital relativiza los códigos deontológicos.

En una investigación con el dilema del niño llorando (J. D. Greene, 2009) el investigador probó que los impulsos iniciales pueden contenerse con un proceso de deliberación que activa una segunda área cerebral. Este es el dilema: Una madre y su hijo están ocultos junto con otras muchas personas en un sótano, en suelo enemigo en situación de guerra, el llanto del niño alertará a los enemigos que si lo oyen matarán a todos los refugiados. ¿Llegaría a matar al niño si fuese necesario? En todos los sujetos se incrementa la actividad en el córtex cingulado anterior. Cuando la respuesta al dilema es: sí, mataría a mi hijo, se eleva la actividad en el córtex prefrontal dorsolateral, sugiriendo un procesamiento adicional que reemplaza la reacción inicial ante lo impactante del dilema.

El otro hallazgo relevante en la investigación es la relación significativa entre la elección deontologista y el sentimiento de culpa, que impide tomar la decisión de dañar al otro en beneficio del colectivo. Esta asociación tiene significación estadística: un patrón de personalidad compulsivo-esquizoide-evitante con depresión-ansiosa. De lo que se infiere que se trataría de una un sentimiento de culpa obsesivo-rumiadora difícil de confrontar y que podría tener matices persecutorios generando ansiedad. La elección deontológica en este caso es un mecanismo de evitación que le determina y le impide un ejercicio libre para elegir. 


\section{Discusión y conclusiones}

¿Basta con una nueva potencialidad para realizar acciones morales distintas? Es condición necesaria pero no es suficiente. La violencia estructural de la cultura coloniza la mente con referentes que justifican la violencia. En Kafka: intento fallido de liberarse del colonizador interno (Bleichmar, 2016) el autor muestra como los intentos individuales de liberación pueden ser fallidos. La psicoterapia tiene como objetivo el análisis de los aspectos más íntimos del impacto de la violencia estructural, pero no aborda la totalidad del fenómeno. El sujeto cambia radicalmente de actitud desde las creencias iniciales fanáticas hasta una perspectiva de realidad compleja. Está más capacitado para la praxis de una moralidad más autónoma. Puede razonar abstractamente y considerar las relaciones de complejidad con un juicio basado en la justicia como valor universal.

Pero la ejecución sigue manteniendo un alto grado de contingencia a las circunstancias de una situación real. Cuando se es agente activo de la acción moral, la ejecución concreta dependerá de la complejidad del vínculo que establezca con el agente pasivo que recibe la acción. Esto es consecuencia del efecto de la dimensión transpersonal que se activa en la relación. La relación caraa-cara es fundamental, como afirma Lévinas. "Soy totalmente solo; así, pues, el ser en mí, el hecho de que existo, mi existir, es lo que constituye el elemento absolutamente intransitivo, algo sin intencionalidad ni relación. Todo se puede intercambiar entre los seres, salvo el existir» (Lévinas, 2000, pp 53 y 54). En esos contextos, de activación compleja de la información, la empatía con las emociones y pensamientos de los demás sobrepasa las opiniones preestablecidas.

Inicialmente el infante humano es amoral, adquiere una conciencia ética a lo largo de su desarrollo, según constatan investigaciones psicoanalíticas, Piaget o Kohlberg (Kohlberg et al., 1997), entre otros. Wellman (2001) afirma que las habilidades socio-cognitivas se desarrollan durante los primeros 5 años de vida y se consolidan en la adolescencia. La investigación psicoanalítica sitúa la terminación de la fase edípica alrededor de los 5 años. Blakemore \& Choudhury, (2006) aportan datos neurocientíficos en la misma línea. Sin embargo, no todo sujeto llega al desarrollo potencial de la conciencia moral. Muchos se quedan fijados en estadios previos y funcionan con una moral inmadura.

Las respuestas deontológicas pueden estar motivadas por la necesidad de conformación social. Un sujeto de la muestra expresó que eligió pasar un día en vez de acostarse y tener una relación sexual porque pensaba o imaginada que era lo aceptable en la sociedad. Everett (2016) hace una investigación que concluye que las personas que hacen juicios deontológicos son preferidas como agentes sociales, porque son percibidos como más morales y de confianza.

Las opciones deontologistas son prevalentes. Efectivamente pueden cumplir una función de contener la potencialidad destructiva de sujetos inmaduros que son la mayoría en la sociedad. Las normas interiorizadas en el superyó freudiano pueden ser útiles. Paradójicamente esta perspectiva es utilitarista, situada en un contexto de contención de la patología. Sin embargo, el freno puede soltarse como pasa en tiempos de guerra en los que destruir al enemigo es un bien. Para la guerra se necesitan sujetos con tendencias internas predispuestas al sadismo. 
El daño a la víctima, parece ser un factor determinante, no el daño objetivo sino la construcción subjetiva de quien elige. Incluso el dilema de la gratificación puede ser percibido como daño a un tercero ausente (la pareja actual o edípica) como si fuese una víctima de una injusticia, y esa percepción determina la elección. Por el contrario, se puede dejar de ver el daño en los casos de genocidio (Castano \& Giner-Sorolla, 2006).

No hacer daño es habitualmente un mecanismo de defensa frente a la retaliación (ser dañado), más que una actitud altruista. Si este no basta aparecen otros: negación, justificación (considerar infrahumanos a los aborígenes australianos citado por Castano), o la escisión. "No hagas a otro lo que no quieras que te hagan a ti» es percibido e interpretado de modo muy diverso según las subjetividades. Algunos sujetos del estudio han recurrido al mismo para no acceder a producir el dolor demandado por su pareja. Algunos sujetos perciben como empatía lo que es un mecanismo defensivo de identificación proyectiva, y eso les lleva a un automatismo deontológico. No hacen daño para no sufrir ellos evitando sentir tanto dolor del otro.

En el polo opuesto carecer de una respuesta empática al dolor de las víctimas podría favorecer elecciones utilitaristas de un modo automático. Incluso consciente, canalizando venganza, centrados en la inmediatez del acto sin proyección de fututo como en los psicópatas.

Algunos sujetos han referido la opción de un autosacrificio antes que hacer daño, tirándose al agua en vez de tirar al moribundo. Puede tener varios significados no explorados en esta investigación; descargar culpa, evitar el castigo (mayor) de otro con un autocastigo controlado; complejo de héroe en la polaridad narcisista-masoquista; un acto de pureza. ¿Hacer el bien para evitar el mal mayor? Algunas de estas disposiciones están en el ideal social que premia, $y$, a veces exige, el autosacrificio.

También se recoge la idea de que el sufrimiento de la víctima (moribundo del naufragio) se comprende como un castigo de la justicia divina a través de un suceso catastrófico: la víctima merece lo que le pasa.

Sachdeva (2015) investiga sobre el significado del autosacrificio en el juicio moral. Sobre el suicidio hay investigadores que afirman que la percepción de daño predice el juicio moral ante el suicidio (Gray, 2014), pero otros consideran que la pureza es más importante que el daño (Rottman, Kelemen, \& Young, 2014).

La percepción de daño se asocia al sistema castigo/recompensa por los actos morales extendida a otras posibles existencias (karma/reencarnación; cielo/infierno). Lo que subyace es la tierra tenebrosa de la ansiedad ante la muerte que la Teoría del Manejo del Terror (TMT) considera clave en la dinámica de los sujetos. Investigadores de la Complutense (Piñuela Sánchez \& Yela García, 2016) han investigado desde la perspectiva (TMT), el efecto de la propia mortalidad (MS) sobre la minimización sentimental (una forma de deshumanización) de los terroristas islamistas, en función de la orientación política de los universitarios estudiados. Concluyen que los resultados apoyan la tesis de que la MS condiciona la actitud más que la posición política.

Experimentar daño en la infancia consteliza un trauma que impacta más allá del dolor, pues frena la construcción de la ética y de la propia estructura 
neurológica. La víctima es, por definición, impotente, la emoción primaria que siempre acompaña a la victimización es el miedo, y las defensas la disociación y la paranoia. En pacientes con trastorno de estrés postraumático se han constatado cambios estructurales en la corteza prefrontal, ACC (cingulado anterior), OFC (orbitofrontal) y amígdala (Francati, Vermetten, \& Bremner, 2007). Áreas comprometidas con el juicio moral. Un estudio reciente con mujeres adultas que experimentaron abusos en la infancia (Nazarov et al., 2016) concluye que la vergüenza, culpa y temor a ser desaprobadas les impedía hacer elecciones utilitaristas. Además, presentaban un déficit de razonamiento altruista asociado a una falta de empatía. Johanna Meehan (1995) expone que supervivientes del incesto, principalmente mujeres, sufren falta de autoconfianza porque su identidad se ha construido sin respeto a su propio cuerpo. W. Reich (1933) fundamentó en la miseria sexual una de las causas del auge del fascismo.

Casi todos tenemos un grado de afectación traumática. Una consecuencia es la confusión entre agresividad y violencia, que es una de las causas profundas de los conflictos, internos y externos. Para Aristóteles «...lo violento (bíaios) es contrario a la naturaleza, y posterior a lo que es conforme a ella. De manera que, si en ningún ser corporal hay un movimiento natural, no habrá movimiento de ninguna clase» (Martínez Guzmán, 2001, p. 209). Lo conforme a la naturaleza es la agresividad. El concepto de fuerza de Hannah Arendt (1998, p.147) se refiere a lo mismo. La violencia social es registrada en términos individuales como terror paralizante. Para la psicoanalista Betty. A. Reardon (1996) el origen de la violencia, que justifica el sexismo y la guerra, es el miedo a la diversidad por el temor a perder el poder. Se origina en la infancia en ambos géneros. El varón se siente vulnerable ante la madre y reacciona con un intento de dominio de lo femenino y cierta fascinación por la guerra. En esta condición se ha perdido el poder comunicativo de actuar concertadamente (Habermas, 1984).

Para cambiar la respuesta moral se necesita actuar tanto en la estructura social como en el sujeto. La psicoterapia actúa en el sujeto individualmente o en grupo (Burlingame \& Layne, 2001).

El cambio en la estructura necesita tanto deconstruir, para lo que la filosofía, la política, la ciencia, aportan ideas y acciones, como aportar planteamientos que propicien deliberación transformadora. Por citar alguno: las propuestas de reconocimiento elaboradas por Axel Honneth (1997) como alternativas para la transformación pacífica de los conflictos o la de Judith Butler quien con una visión política de deconstrucción antiesencialista, recoge ideas de Austin; de la visión construccionista de la sexualidad de Foucault; de Jacques Derrida; del psicoanálisis lacaniano; de Simone de Beauvoir, para su propuesta performativa de género (Acosta, 2010).

\section{Conclusiones}

Esta investigación se ha diseñado desde una perspectiva consiliente, y, por ello, tiene interés compartirla en la comunidad científica. "A menudo decir algo 
producirá ciertas consecuencias o efectos sobre los saberes, pensamientos o acciones del auditorio o de quien emite la expresión... un acto performativo» (Austin, 1971). Algunos sujetos comunicaron que interaccionar con el material de la investigación les supuso tomar contacto con actitudes propias que les eran desconocidas hasta entonces, que es la condición inicial para cambios.

La articulación de análisis cuantitativo y cualitativo es poco frecuente en las investigaciones y, en esta investigación, se ha evidenciado su potencial generador de significado.

Es interesante expandir interés en indagar sobre las estructuras (cajas negras) que dan sentido. Tal y como dice Edgar Morin (1994), el pensamiento complejo está animado por la aspiración a un saber no parcelado, no dividido, y no reduccionista.

Los resultados avalan la propuesta de que es útil incorporar una elaboración de la moralidad antes del alta en las psicoterapias. Explorando con el paciente su conciencia moral, su relación con el otro en el contexto político social, y la muerte propia.

Es una investigación incompleta que apunta datos para reflexionar, repensar ideas y paradigmas previos.

En investigaciones futuras sería conveniente repensar los dilemas. Cabe dudar sobre si el criterio de cantidad es el más adecuado, ¿salvar seis personas en vez de una es más utilitarista? "Un hombre libre en nada piensa menos que en la muerte, y su sabiduría no es una meditación de la muerte, sino de la vida" (Baruch Espinosa en su Ética). Quizás esa sabiduría valorará la utilidad de otra forma por ejemplo asociada a la cualidad. Como mínimo incorporar escenarios más intersubjetivos contemplando el fenómeno de la transferencia.

El innatismo es conveniente sea operativizado en investigaciones futuras. Aportaciones del psicoanálisis sobre informaciones prepersonales en forma de precogniciones, fantasías inconscientes, y arquetipos colectivos (León-Río, 2009) que prefiguran la capacidad simbólica y son organizadores psíquicos para la construcción de la conciencia moral. De acuerdo con Carl Jung (2002) en la relación con el arquetipo se puede sostener que se necesita la aceptación de acciones malas, incluso espantosas, que la vida nos invita a cometer en aras de un propósito mayor (Hannah, 2010, p. 31). El sujeto de la acción sufrirá en su propia alma, sin que un sentimiento de culpa, bloquee la acción o le paralice en la inercia de la negación de la vida, o le conduzca a la autocompasión. El sufrimiento es el requisito de veracidad. En términos psicológicos un sujeto debería sacrificar su yo para seguir evolucionando. Esto significa la necesidad de matar el vínculo del yo con un narcisismo excluyente del otro lo que es condición para la paz positiva. Quizás entonces pudiera ser real lo que afirma Sartre sobre la libertad «... el hombre no tiene naturaleza, no tiene una esencia, por lo que es libre y es lo que él mismo ha decidido ser». Algunos sujetos de la investigación emitieron respuestas cercanas a esta posición.

La praxis moral también está condicionada por la cognición integrada del proceso terciario (Arieti, Silvano, 1976; Fiorini, Hector J., 1995; Washburn, 1997, 1999). A ese estado, mucho más complejo de funcionamiento, se llega mediante la integración de las diversas facultades y dimensiones humanas, personales y 
transpersonales. Es un área a explorar e integrar en las investigaciones sobre la conciencia moral. Kohlberg ya había adelantado un estado moral transconvencional con su etapa cósmica (Kohlberg, L y Power, F., 2012), apuntando, también, más allá de un desarrollo secuencial de las etapas, que los sujetos pueden tener distintos niveles de desarrollo moral no integrados en una coherencia, ya que disocian los distintos niveles de desarrollo moral aplicando uno $u$ otro en según qué áreas de su existencia. Otros autores han aportado revisiones de la etapa postconvencional de Kohlberg (Rest, James, Muriel Bebeau, Darcia Narváez, 1999).

Las investigaciones necesitan realizar algún ensayo clínico para diseñar alguna intervención que pueda resultar efectiva para transformar la conciencia moral y actitudes hacia la paz de los sujetos y que pueda ser implementada por sujetos que no sean psicoterapeutas.

\section{Bibliografía}

Acosta, C. A. D. (2010). Judith Butler y la teoría de la performatividad de género. Revista de educación y pensamiento, (17), 85-95. Arendt, H. (1998). Sobre la violencia. En Crisis de la república. Madrid: Taurus.

Arieti, Silvano. (1976). La Creatividad: La Síntesis Mágica. Fondo de Cultura Económica.

Austin, J. L. (1971). Palabras y Acciones Como Hacer Cosas Con Palabras. Buenos Aires: Paidós.

Bentham, J. (1983). Deontology; together with a table of the springs of action; and the article on utilitarianism (The Collected Works of Jeremy Bentham). Oxford, England: Oxford University Press. (Original work published 1879).

Burlingame, G. M., \& Layne, C. M. (2001). Group-based interventions for trauma survivors: Introduction to the special issue. Group Dynamics: Theory, Research, and Practice, 5(4), 243-245.

Castano, E., \& Giner-Sorolla, R. (2006). Not Quite Human: Infrahumanization in Response to Collective Responsibility for Intergroup Killing. Journal of Personality and Social Psychology, 90(5), 804-818.

Cunyat Agut, M. (2016). Darcia Narváez (2014) Neurobiology and the development of human morality: evolution, culture and wisdom New York: WW Norton \& Co., 393 pp. Anuari de Psicologia, Monogràfic Jove 2, 211-212.

Elster, J., \& Grapes, S. (1982). Utilitarianism and the genesis of wants.

Everett, M. J., Jim A. C. .. Pizarro, David A. .. Crokett. (2016). Inference of Trustworthiness from Intuitive Moral Judgments. Intuitive moral judgments and partner choice. Journal of Experimental Psychology: General, 772-787(145.6), Inglés.

Fiorini, Hector J. (1995). Formaciones de procesos terciarios Una tópica del psiquismo creador. Paidós.

Francati, V., Vermetten, E., \& Bremner, J. D. (2007). Functional neuroimaging studies in posttraumatic stress disorder: review of current methods and findings.

Depression and Anxiety, 24(3), 202-218.

Garaigordobil Landazabal, M. (2008). Evaluación del programa "Una sociedad que construye la paz-Bakea eraikitzen ari den gizartea». Vitoria-Gazteiz: Eusko Jaurlaritzaren Argitalpen Zerbitzu Nagusia.

Garaigordobil, M. (2011). LAEA. Listado de adjetivos para la evaluación del autoconcepto en adolescentes y adultos. Madrid: Pirámide.

La psicoterapia en la transformación de la praxis moral y las actitudes hacia la paz. Mikel Garcia Garcia 
Glaser, B. (2005). Grounded theory perspective III: theoretical coding. Chicago: Sociology Press.

Gray, K. (2014). Harm concerns predict moral judgments of suicide: Comment on Rottman, Kelemen and Young (2014). Cognition, 133(1), 329-331.

Gray, K., Waytz, A., \& Young, L. (2012). The Moral Dyad: A Fundamental Template Unifying Moral Judgment. Psychological Inquiry, 23(2), 206-215.

Greene, J. D. (2009). The cognitive neuroscience of moral judgment. The Cognitive Neurosciences IV.

Greene, J. D., Nystrom, L. E., Engell, A. D., Darley, J. M., \& Cohen, J. D. (2004). The neural bases of cognitive conflict and control in moral judgment. Neuron, 44(2), 389400.

Greene, J. D., Sommerville, R., Nystrom, L., Darley, J., \& Cohen, J. (2001). An fMRI investigation of emotional engagement in moral judgment. Science (New York, N.Y.), 293(5537), 2105-2108.

Habermas, J. (1984). El concepto de poder en Hannah Arendt. En Perfiles filosóficopolíticos. Madrid: Taurus.

Haidt, J., \& Joseph, C. (2007). The moral mind: How 5 sets of innate moral intuitions guide the development of many culture-specific virtues, and perhaps even modules. En P. Carruthers, S. Laurence y S. Stich (Ed.), The Innate Mind (Vol. 3, pp. 367-391). New York: Oxford.

Hannah, B. (2010). El Viaje Interior. Ensayos sobre psicología junguiana. Mexico, D.F.: Fata Morgana.

Henwood, K. (2004). Reinventing validity: Reflections on principles and practices from beyond the quality-quantity divide. En D. Todd Z. .. Nerlich, B. .. Mckeown, S. \&. Clarke (Ed.), Mixing methods in psychology (pp. 37-57). Hove: Psychology Press.

Henwood, K. (2008). Qualitative research, reflexivity and living with risk: Valuing and practicing epistemic reflexivity and centering marginality. Qualitative research in psychology, 5(1), 45-55.

Honneth, A. (1997). La lucha por el reconocimiento. Por una gramática moral de los conflictos sociales (Crítica). Barcelona.

Jung, C. G. (2002). Los arquetipos y el inconsciente colectivo (Obra completa, Vol. 9/1). Madrid: Trotta.

Kant, I. (1979). Crítica de la razón pura. México: Porrúa.

Kant, I. (2008). Fundamentación para una Metafísica de las Costumbres. Madrid: Alianza (Trabajo original Publicado en 1785).

Kimsey, W. D., \& Fuller, R. M. (2003). Conflictalk: An instrument for measuring youth and adolescent conflict management message styles. Conflict Resolution Quarterly, 21(1), 69-78.

Koenigs, M., Kruepke, M., Zeier, J., \& Newman, J. P. (2012). Utilitarian moral judgment in psychopathy. Social Cognitive and Affective Neuroscience, 7(6), 708-714.

Kohlberg, L. (1982). Moral stages and moralization. A cognitive developmental approach. Infancia y Aprendizaje, 5(18), 33-51.

Kohlberg, L. (1984). Psicología del desarrollo moral. Bilbao: Desclée de Brouwer (1992).

Kohlberg, L., Power, F. C., \& Higgins, A. (1997). La educación moral según Lawrence Kohlberg. Gedisa Barcelona.

Kohlberg, L y Power, F. (2012). Desarrollo moral, pensamiento religioso y la cuestión de una Séptima Etapa. Revista Postconvencionales, No. 5-6, 163-210.

León-Río, B. (2009). Arquetipos e inconsciente colectivo en las artes plásticas a partir de la psicología de C. G. Jung. Arte, individuo y sociedad, (21), 37-50. Lévinas, E. (2000). Ética e infinito (A. Machado Libros, S.A). Madrid.

MacIntyre, A. (1981). After virtue. Londres: A\&C Black Publishers (2013). 
MacLean, P. D. (1990). The triune brain in evolution: Role in paleocerebral functions. New York: Plenum Publishing Corporation.

Martínez Guzmán, V. (1997). Reconstruir la paz doscientos años después. Una filosofía transkantiana para la paz. En La paz en Kant doscientos años después (pp. 119134). Valencia: Nau Llibres.

Martínez Guzmán, V. (2001). Filosofía para hacer las paces. Icaria.

Mayntz, R., Holm, K., Hübner, P., \& Muñiz, J. N. (1969). Introducción a los métodos de la sociología empírica. Madrid: Alianza Editorial (1993).

Meehan, J. (1995). Feminists read Habermas, Gendering the Subject of Discourse,. New York: Routledge.

Mill, J. S. (1989). El utilitarismo. Madrid: Alianza (Trabajo original en 1863).

Millon, T. (2007). MCMI Inventario Clinico Multiaxial. Manual. Madrid: TEA.

Moral de la Rubia, J., García-Cadena, C. H., \& Antona-Casas, C. J. (2012). Traducción y validación del Inventario Balanceado de Deseabilidad Social. Revista de Psicología GEPU, 3(2), 54-72.

Moral De la Rubia, J., Medina, J. L. V., \& Bravo, B. G. A. (2011). Creación de una Escala para Medir Paz Personal. REMA Revista electrónica de metodología aplicada, 16(2), 1-14.

Narvaez, D. (2014). Neurobiology and the Development of Human Morality: Evolution, Culture, and Wisdom. New York: WW Norton \& Company.

Nazarov, A., Walaszczyk, V., Frewen, P., Oremus, C., Lanius, R., \& McKinnon, M. C. (2016). Moral reasoning in women with posttraumatic stress disorder related to childhood abuse. European Journal of Psychotraumatology, 7. Paulhus, D. L. (1988). Manual for the Balanced Inventory of Desirable Responding (BIDR-6). University of British Columbia.

Piñuela Sánchez, R., \& Yela García, C. (2016). Mortality salience, political orientation and minimization of terrorists' secondary emotions. Psicothema, 28(1).

Reardon, B. (1996). Sexism and the war system. Syracuse University Press.

Regan, D. (1980). Utilitarianism and Co-operation. Oxford University Press.

Reich, Wilhelm. (1933). Psicología de masas del fascismo (1973). Barcelona: Roca.

Rest, James, Muriel Bebeau, Darcia Narváez. (1999). Postconventional Moral Thinking. A NeoKohlbergian Approach. New Jersey: Lawrence Erlbaum Associate.

Rottman, J., Kelemen, D., \& Young, L. (2014). Purity matters more than harm in moral judgments of suicide: Response to Gray (2014). Cognition, 133(1), 332-334.

Sachdeva, S., Iliev, R., Ekhtiari, H., \& Dehghani, M. (2015). The Role of Self-Sacrifice in Moral Dilemmas. PLoS ONE, 10(6), e0127409.

Scanlon, T. M. (1982). Contractualism and utilitarianism. En A. K. Sen \& B. A. O. Williams (Ed.), Utilitarianism and beyond (pp. 103-110). England: Cambridge University Press.

Scanlon, T. M. (1998). What we owe to each other (Vol. 66). Cambridge, MA: Belknap Press of Harvard University Press. Recuperado a partir de

Skitka, L. J., \& Sargis, E. G. (2006). The Internet as Psychological Laboratory. Annual Review of Psychology, 57(1), 529-555.

Strawson, P. F. (1995). Libertad y resentimiento y otros ensayos (Vol. 37). Grupo Planeta (GBS).

Washburn, M. (1997). El ego y el fundamento dinámico. Barcelona: Kairós.

Washburn, M. (1999). Psicologia Transpersonal, en una perspectiva psicoanalitica. Barcelona: Los libros de La Liebre de Marzo. 


\section{Apéndice 1}

\section{Cuestionario de Paz o Equilibrio en La Vida (CPEV-20)}

felicidad-afabilidad; honestidad-responsabilidad; iniciativa-independencia; serenidad-

tolerancia; autoestima-equilibrio

Inventario Balanceado de Respuestas Socialmente Deseables

Autoengaño; Manejo Impresión

Cuestionario de estilos de mensaje en el manejo del conflicto

Manejo del conflicto enfocado en sí mismo: impositivo; Manejo del conflicto enfocado en los otros: evitativo; Manejo del conflicto enfocado en el problema: cooperativo.

The Attitudes About Conflict Scale EAC

Capacidad de resolución positiva del conflicto.

LAEA. Listado de adjetivos autoconcepto

Físico; Social; Emocional; Intelectual

\section{Apéndice 2}

Dilemas morales

\section{Gratificación}

Un día tienes la oportunidad de elegir entre acostarte una noche con una persona que es famosa y que deseas sexualmente con el compromiso de mantener ese hecho en secreto o pasar con dicha persona un día de modo que además de que seáis vistos en compañía puedas hablar de ello con quien quieras. ¿Qué elegirías?

\section{Demanda causar dolor}

Como seguramente sabrás, las personas masoquistas gozan sexualmente sintiendo dolor o humillación. En caso de que convivan con una persona que posea rasgos o tendencias sádicas, sus relaciones son satisfactorias para los dos miembros de la pareja, y las repercusiones de ese dolor placentero son asumibles, en términos generales, para ambos.

El problema sexual aparece cuando uno de los dos miembros de la pareja no goza con tales actos. Por ejemplo, un individuo que conviva con una persona masoquista se ve en el dilema de causar dolor (algo que en sí mismo le repugna moralmente) para que su pareja goce.

Si tú te encontraras en una situación de ese tipo, ¿accederías a causar dolor a la persona a la que quieres o decidirías romper con ella a pesar de tu amor por creer que estás cometiendo acciones inmorales?

\section{Tirar al moribundo}

El hombre de pie sostiene a un moribundo. Si tu estuvieras en esa situación ¿̇lo tirarías al agua?

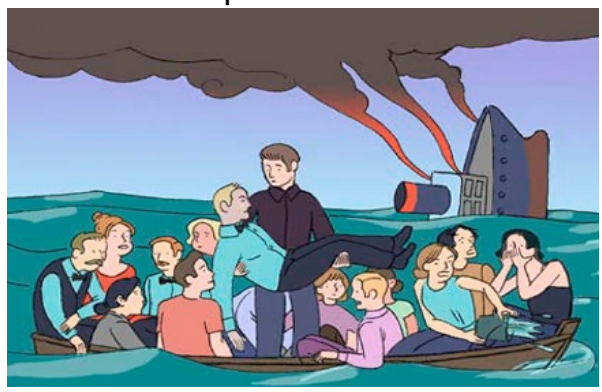

La psicoterapia en la transformación de la praxis moral y las actitudes hacia la paz. Mikel Garcia Garcia 


\section{Perdón}

La emoción es una reacción psíquica que altera el equilibrio y el comportamiento racional de cualquier persona. Sin embargo, existen individuos capaces de dominarla, y nunca (o casi nunca) pierden el control de sus actos, mientras otros, en cambio, se dejan llevar por ella y realizan, bajo su efecto, acciones de las que posteriormente se arrepienten.

Imagínate que tu pareja, arrastrada por la emoción y el cariño que profesa a un antiguo/a amigo/a, y ante la insistencia de éste/a, apelando al afecto que aún existe entre los dos, comete un acto de infidelidad para contigo.

¿Lo/a perdonarías por el hecho de haber actuado con una gran carga emotiva? ¿O no lo harías por creer que podía haber controlado su emoción antes de realizar un acto que traicionaba vuestra mutua confianza?

\section{5 y 6 . Excluidas de esta investigación.}

\section{Autorizar inseminación}

Suponte que eres juez y en tu juzgado entra una demanda de una mujer que solicita utilizar el semen de su marido muerto para tener un hijo mediante inseminación artificial. Tú ignoras cuál era la voluntad del muerto al congelar su semen (podría ser, por ejemplo, para tener descendencia con otra mujer diferente a la suya), ya que sólo tienes como referencia la palabra de su esposa, sin que puedas contrastarla con ninguna otra.

Se te plantea el dilema de autorizar o denegar la inseminación artificial, basándote únicamente en razones éticas, ya que la ley no contempla específicamente un caso como éste. ¿Qué harías y cuál sería tu justificación?

\section{Altruismo/propio interés.}

Imagínate que buscas trabajo y te contratan en una industria que se dedica a la elaboración de productos químicos. Al cabo de los meses compruebas, por tu propia experiencia, que la empresa no respeta la legislación medioambiental vigente y está contaminando las aguas de un río con los residuos de una sustancia química que pasa desapercibida a simple vista y cuyos efectos no pueden ser comprobados a corto plazo, aunque sí a largo.

Esto te plantea un dilema de difícil resolución: ¿debes denunciar a la empresa por causar contaminación, o debes guardar silencio para garantizar tú puesto de trabajo e incluso el de tus compañeros (te podrían despedir, o si la multa y los costes para adaptarse a la legislación ecologista fuesen muy altos la empresa podría cerrar)? ¿Qué podría más en este caso concreto: tú interés ecológico o tu interés personal por el trabajo? 